\title{
A Survey of Underwater Wireless Communication Technologies
}

\author{
Camila M. G. Gussen, Paulo S. R. Diniz, Marcello L. R. Campos, \\ Wallace A. Martins, Felipe M. Costa, and Jonathan N. Gois
}

\begin{abstract}
The increasing exploitation of natural resources under water, particularly in the sea, has ignited the development of many technological advances in the domains of environmental monitoring, oil and gas exploration, warfare, among others. In all these domains, underwater wireless communications play an important role, where the technologies available rely on radiofrequency, optical, and acoustic transmissions. This paper surveys key features inherent to these communication technologies, putting into perspective their technical aspects, current research challenges, and to-be-explored potential.
\end{abstract}

\section{INTRODUCTION}

Underwater wireless communications present new and distinct challenges when compared to wired and wireless communications through the atmosphere, requiring sophisticated communication devices to achieve relatively low transmission rates, even over short distances. Indeed, the underwater environment possesses a number of distinguishing features that make it unique and rather different from terrestrial radio propagation where traditional communication systems are deployed. Under water, several phenomena may influence communications, such as salt concentration, pressure, temperature, amount of light, winds and their effects on waves, just to mention a few [1], [2].

Despite all challenges, wireless communications certainly play an important role in practical underwater systems. Monitoring different phenomena in underwater environment is relevant in many different applications, such as oil and gas exploration, coastal security, environmental impact surveillance, navigation, and ocean-pollution control [3]. Specific examples include transmission of data among devices, such as AUV (autonomous underwater vehicle) to AUV and buoy to AUV, particularly those employing wireless links. In fact, an entire underwater wireless communication network could

C. M. G. Gussen, P. S. R. Diniz, M. L. R. Campos, and W. A. Martins are with the Department of Electronics and Computer Engineering and with the Program of Electrical Engineering, COPPE/Poli, Federal University of Rio de Janeiro, Rio de Janeiro, RJ, 68504, Brazil. E-mails: \{camila.gussen,diniz,campos,wallace.martins\}@smt.ufrj.br.

F. M. Costa is with the Systems Integration group in GE Global Research,Rio de Janeiro, RJ, e-mail: F.Costa@ge.com.

J. N. Gois is with the Federal Center of Technological Education (CEFET/RJ-UnED-NF), Nova Friburgo, RJ, and with the Program of Electrical Engineering, COPPE/Poli, Federal University of Rio de Janeiro, Rio de Janeiro, RJ, e-mail: jonathan.gois@ cefet-rj.br.

The authors wish to thank the Brazilian research, development, and innovation agencies CAPES (Prodefesa, 23038.009094/2013-83) and FINEP (01.13.0421.00), the Brazilian Navy Secretary of CT\&I/SECCTM, the Institute of Sea Studies Admiral Paulo Moreira/IEAPM, and the GE Global Research Center in Rio de Janeiro, Brazil, for their support of this work.

Digital Object Identifier: 10.14209/jcis.2016.22 be deployed through several configurations involving floating devices, AUVs, seafloor-attached sensors or processing towers, submarines, or ships. Although there are plenty of opportunities, one can find very few off-the-shelf solutions for reliable and economically viable underwater wireless communications, a trend that will certainly change in the near future given the current growing demand.

There are three main technologies available for underwater wireless transmissions. One technology is radio-frequency (RF) communication, which features high data throughput at short range and suffers from mild Doppler effect. Other technology is optical transmission, preferably in blue-green wavelength, which requires line-of-sight positioning. Another technology, which is the most employed one, is acoustic communication. This latter technology is the one that allows the longest range of communication, but achieves low throughput, is highly impaired by Doppler effects, ${ }^{1}$ and is affected by large delay spread that leads to severe intersymbol interference [4]. In all these technologies, it is important to consider both the implementation costs associated with a target data throughput for a prescribed communication range, as well as the relative transmission power that might lead to environmental impacts such as interference with marine life. ${ }^{2}$

The aim of this paper is to survey the main features inherent to each underwater wireless communication technology. The paper also discusses how signal processing brings about possible solutions to current practical challenges to improve the capability of data communication in the underwater environment. The target audience includes communication and signal processing researchers/students as well as practicing engineers. The presentation, therefore, follows a path that is commonplace among those intended readers, focusing on objectively presenting the main principles of the subject matter. The idea is to provide researchers, students, and engineers with a clear picture of the technical aspects, open research problems, and related business potential of this fruitful and still promising communication field.

The paper is organized as follows: Section II provides the big picture. Sections III, IV, and V discuss issues related to RF, optical, and acoustic communications, respectively. Section VI addresses the formation of underwater wireless networks. Section VII briefly compares the communication

\footnotetext{
${ }^{1}$ A description of Doppler effect is provided in Subsection V-C.

${ }^{2}$ It is worth mentioning that there is widespread awareness of these issues among both civilians and the military. However, underwater modems typically use much less power than, e.g., military sonar systems or geoacoustic surveying systems.
} 
technologies.

\section{UNDERWATER WIRELESS COMMUNICATION OVERVIEW}

An illustrative example of an underwater environment capitalizing on multiple communication technologies is depicted in Figure 1. Signal communication in such environment might include several possibilities such as links from land to satellite, then to buoy ship and/or oil platform. It is also possible to exchange data through RF antennas located at floating devices and land stations. Communication devices might be attached to floating structures to allow the exchange of information with stations placed underwater. In the water environment, it is possible to deploy numerous different types of communication nodes consisting of AUV's, local area wireless and wired networks. Some devices might be anchored or attached to the seafloor.

In such flexible communication environment, it is possible to establish a software-defined network (SDN) where a large number of communication devices, each one with its inherent features, can exchange data. Considering that wireless links are highly desirable for underwater applications, proper knowledge of the physical constraints on information transmission over the physical layer must be acquired.

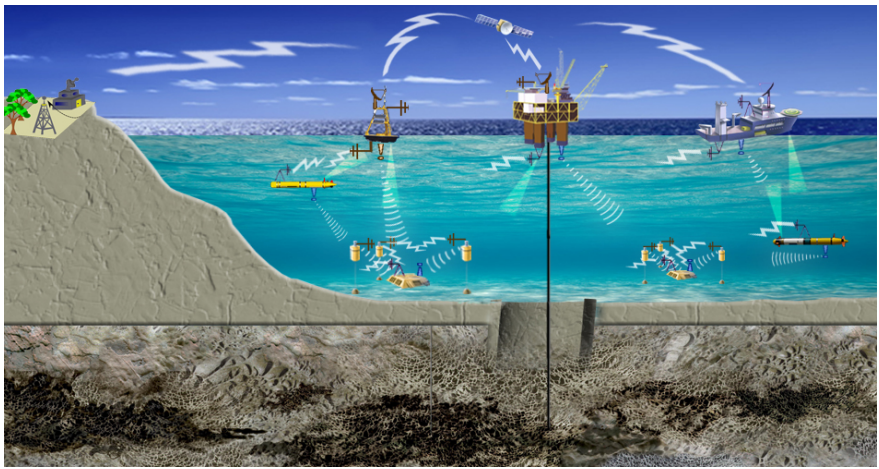

Figure 1. Scenarios of multiple communication technologies: stations placed underwater can exchange information with buoy ship and/or oil platform using wireless or wired system. These devices can transmit data to a satellite or to land.

\section{RF COMMUNICATIONS}

From the physics viewpoint, for frequency ranges employed by mobile services, TV, radio, and satellite communications, the seawater is highly conductive, thus seriously affecting the propagation of electromagnetic waves. As a result, it is not easy to establish communication links for distances beyond $10 \mathrm{~m}$ in the ocean [2] in both very- and ultra-high frequency ranges (VHF and UHF, respectively), or even in high frequencies. At lower frequencies, namely at extremelyand very-low frequency ranges (ELF and VLF, respectively), the electromagnetic-wave attenuation can be considered low enough to allow for reliable communications over several kilometers. Unfortunately, these frequency ranges from $3 \mathrm{~Hz}$ to $3 \mathrm{kHz}$ and from $3 \mathrm{kHz}$ to $30 \mathrm{kHz}$ are not wide enough to enable transmissions at high data rates. Despite being used in naval [5] and environmental applications [6], performing communication in ELF and VLF frequency ranges has operational and financial difficulties, since the equipment is large, expensive and requires high power.

Another characteristic of underwater RF signals is that they can travel through several paths: the signal can cross the water-air boundary and can propagate through the seabed, as illustrated in Figure 2. Hence, it might be possible to use these multiple paths to increase the signal propagation distance in shallow water, and, as a consequence, a submerged station can transmit information to an onshore station [7]. In this case, the signal traversing the seabed or the air might suffer lower attenuation than the signal that propagates only in the water. In addition to these features, the RF signal also suffers Doppler effect. Despite being not so pronounced as in the acoustic case, this channel feature needs to be properly considered and treated.

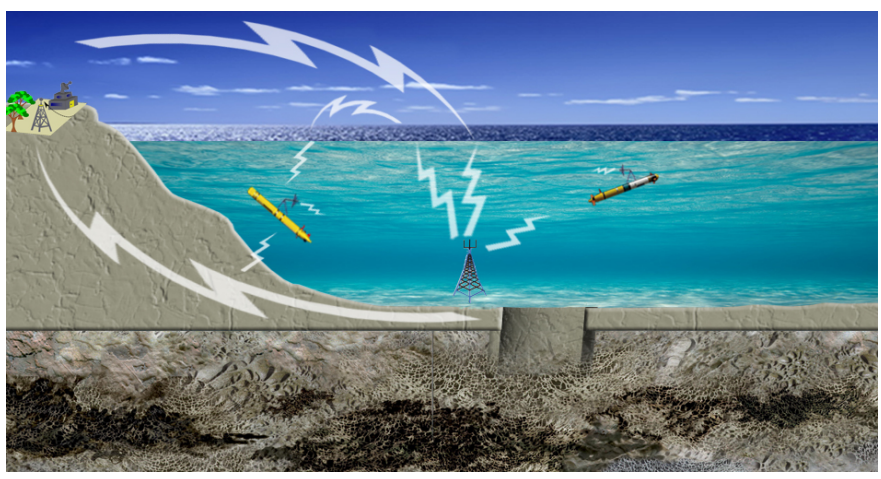

Figure 2. Possible multipath propagation of a RF signal in shallow water environment.

The propagation of the RF signal depends on environmental conditions, such as salinity and temperature, and is frequency dependent. Let $\alpha(f)$ represent the channel attenuation per meter, whose typical model in seawater is [8]

$$
\alpha(f)=\sqrt{\pi \sigma \mu_{0}} \sqrt{f}=\kappa \sqrt{f},
$$

where $f$ represents the RF (carrier) signal frequency in Hertz, $\sigma$ represents the water conductivity in Siemens per meter, and $\mu_{0} \approx 4 \pi \cdot 10^{-7} \mathrm{H} / \mathrm{m}$ is the vacuum permeability. The conductivity is a function of salinity and temperature, being around $4.3 \mathrm{~S} / \mathrm{m}$ for seawater, whereas in fresh water it is in the range of 0.001 to $0.01 \mathrm{~S} / \mathrm{m}$. As a result, it is expected that the attenuation of RF signal be higher in seawater than in fresh water, considering that the higher conductivity of the seawater has more impact on the attenuation of the electromagnetic field. The permeabilities of seawater and fresh water are approximately the same. The distinct values of conductivity show the relevance of salinity in the propagation of RF signal. Thus, the main aspect to be considered to characterize the wireless channel for RF transmission is the salinity of the water.

A typical channel model transfer function is described by

$$
H(f)=H_{0} \mathrm{e}^{-\alpha(f) d} \mathrm{e}^{-\mathrm{j} \theta(f)},
$$

with $H_{0}$ being the DC channel gain, $\theta(f)$ being the channel phase, and $d$ representing the distance between transmitter 
and receiver. For a fixed frequency, the channel magnitude response decreases exponentially with distance. Figures 3(a) and 3(b) illustrate variations of the magnitude of the channel response with respect to frequency and distance, considering fresh water and seawater, respectively. The attenuation for seawater is always higher than for fresh water for all distances and frequencies. Moreover, lower frequency and distance leads to less attenuation for all water types. It is worth mentioning that the channel model of Eq. (2) does not consider spreading loss due to the simplified plane-wave model. Besides, even if we were to consider a spherical wave model, the attenuation loss would dominate over the spreading loss due to the high conductivity of the medium: the first increases exponentially with respect to the propagation distance, whereas the second increases proportionally to a power of the propagation distance. Indeed, this source of attenuation dominates all other losses that actually occur in practice.

Table I presents the propagation distance for a signal attenuation of $50 \mathrm{~dB}$. Considering seawater, when using frequencies in the order of $\mathrm{MHz}$ the propagation distance is on the order of centimeters. Despite the short propagation distances the RF technology can be useful in some specific applications, as for example in data transfer between devices in deep water, without mechanical coupling.

\section{A. Noise in Underwater RF Communications}

RF propagation and noise models in underwater environments are not widely discussed in the open literature. One of the few exceptions is the work of [9] suggesting that the environmental noise follows a probability density function similar to Gaussian distribution with zero mean.

\section{B. RF Transducers}

The technology employed for transducers is the same as the one adopted for wireless communications in the air, i.e., antennas. If the employed frequency ranges are ELF and VLF, there is the requirement for large receiving antennas, which can hinder the applicability of RF technology in some cases. Moreover, all the equipment must be properly encapsulated for operation in the underwater environment.

\section{Main Issues in Underwater RF Communications}

The underwater RF communication is heavily affected by propagation loss that should be estimated accurately in application-sensitive operations, such as data retrieval and storage, and power transfer to underwater equipment. In addition, the RF signal faces severe frequency selective channels and it is affected by environmental noise. The channel and noise estimations should be reliable so that channel capacity can be approached. For that the use of multicarrier transceivers with possible channel state estimation and channel loading can be the solution.

Therefore, RF technologies possess severe constraints on data rates and on propagation distances. These are the main reasons for the small number of products using RF communication technology so far. Nonetheless, there are some applications in which alternative technologies based on acoustic or optical transmissions are not viable solutions. For example, one of the most suitable technologies for monitoring seabed sediments in order to control coastal erosion is through the deployment of a sensor network that can exchange information through RF signals [7].

\section{OPTICAL COMMUNICATIONS}

The main difference between RF and optical propagation in seawater is the medium behavior: the water is seen as a conductor for RF and as a dielectric for optical propagation. The explanation for this phenomenon lies in the plasma frequency, whose value determines the range of frequencies for which the medium behaves as a conductor or as a dielectric. The seawater changes from conductor to dieletric at frequencies around $250 \mathrm{GHz}$ [2]. As in dieletric medium the electromagnetic wave has lower attenuation than in conductor medium, the optical technology can provide higher data rates as compared to RF, for a propagation range limited to tens of meters. As the speed of light is around 4 to 5 orders of magnitude larger than the speed of propagation of acoustic waves in fluids, the Doppler spread and its effects are almost negligible in optical wireless communications.

The propagation distance of optical signals depends on the frequency range. The blue-green optical window has lower propagation attenuation and this knowledge has been used for improving blue-green sources and detectors [10], [11]. Optical communications usually require line-of-sight between transmitter and receiver [12], which entails some sort of direction tracking to maintain the communication link.

Considering the effects of environmental conditions, water has two important features that affect light propagation: inherent optical properties (IOPs) and apparent optical properties (AOPs). Inherent optical properties depend only on the medium (water), whereas apparent optical properties depend on the light source characteristics, e.g., if the laser (light amplification by stimulated emission of radiation) source produces collimated or diffuse rays [13]. For optical underwater wireless communication, IOP is more relevant.

The two main IOPs are the spectral absorption coefficient and the spectral volume scattering function [14]. Absorption is the process that transforms electromagnetic radiation into heat, i.e., the energy that would be re-emitted is absorbed. Absorption occurs at chlorophyll in phytoplankton, at colored dissolved organic matter (CDOM), at water molecules, and at dissolved salts in water [13]. The absorption effect can be taken into consideration through the parameter $a(\lambda)$ [15], named spectral absorption coefficient (in $\mathrm{m}^{-1}$ ), with $\lambda$ being the wavelength.

The direction of the photons change due to scattering. Scattering can be originated by salt ions in pure water and by particulate matter [13]. Scattering by objects smaller than the light wavelength is described by the Rayleigh model, whereas scattering by objects greater than the light wavelength is described by Mie theory [2]. The spectral volume scattering coefficient is denoted herein as $b(\lambda)$ [15].

The beam attenuation coefficient is related to the total energy that is lost due to absorption and scattering, and is 


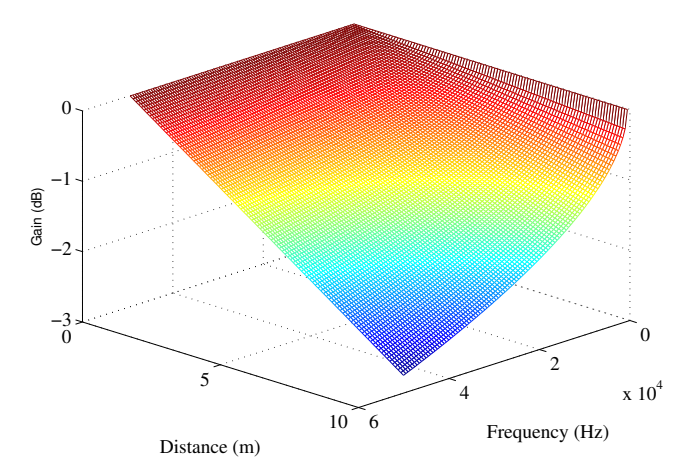

(a) Channel gain versus frequency and distance - Fresh water.

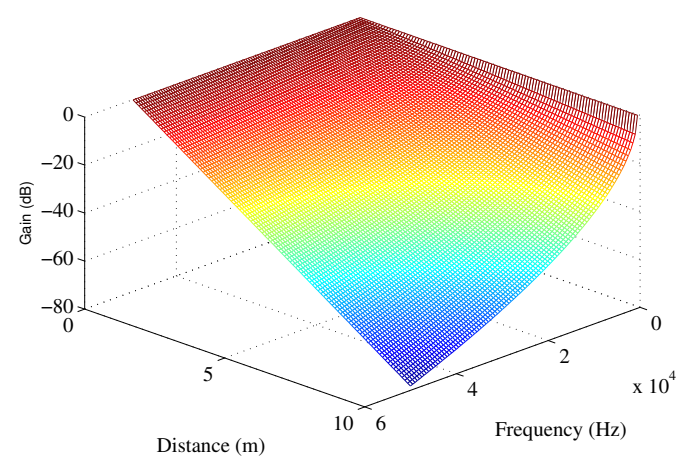

(b) Channel gain versus frequency and distance - Seawater.

Figure 3. Channel properties for RF communications.

Table I

PROPAGATION DiSTANCE IN METERS FOR A SIGNAL ATTENUATION OF 50 DB

\begin{tabular}{|c|c|c|c|c|c|c|c|}
\cline { 2 - 8 } \multicolumn{1}{c|}{} & \multicolumn{9}{c|}{ Frequency } \\
\cline { 2 - 8 } \multicolumn{1}{c|}{} & $10 \mathrm{~Hz}$ & $10^{2} \mathrm{~Hz}$ & $10^{3} \mathrm{~Hz}$ & $10^{4} \mathrm{~Hz}$ & $10^{6} \mathrm{~Hz}$ & $10^{7} \mathrm{~Hz}$ & $10^{9} \mathrm{~Hz}$ \\
\hline Seawater & $4.4 \times 10^{2}$ & $1.4 \times 10^{2}$ & $4.4 \times 10^{1}$ & $1.4 \times 10^{1}$ & $1.4 \times 10^{0}$ & $4.4 \times 10^{-1}$ & $4.4 \times 10^{-2}$ \\
\hline Fresh water & $2.9 \times 10^{4}$ & $9.2 \times 10^{3}$ & $2.9 \times 10^{3}$ & $9.2 \times 10^{2}$ & $9.2 \times 10^{1}$ & $2.9 \times 10^{1}$ & $2.9 \times 10^{0}$ \\
\hline
\end{tabular}

defined as [14], [16], [17]:

$$
c(\lambda)=a(\lambda)+b(\lambda)
$$

Many applications employ the backscattering coefficient, $b_{\mathrm{b}}(\lambda)$, which is part of the spectral volume scattering coefficient $(b(\lambda))$ related to the amount of light that returns to the transmitter. This coefficient can be used to estimate water quality: the knowledge of the water turbidity can be important to the design of smart transmitters, which are able to change transmission power and data rate accordingly [16].

To assess the environmental conditions that affect specifically optical communications, the water has been classified in different ways. According to [2], Jerlov water type includes three main classes: clearest water, intermediate water, and murkiest water. Clearest water can be found in Mid-Pacific and Atlantic, whereas intermediate water is typical in the Northern Pacific ocean. The murkiest water is typical of the North Sea and Eastern Atlantic. Another classification that is commonly considered in the literature [14], [18], divides the water into four types: pure seawater, clear ocean water, coastal ocean water, and turbid harbor and estuary water. In pure seawater, the major loss is due to absorption, while in clear ocean water there is also a scattering loss due to a higher concentration of particles in comparison with pure seawater. Coastal ocean water has even higher concentration of particles that affects scattering and absorption, while turbid harbor and estuary water have the highest concentration of particles. Table II presents typical values for the coefficients $(c(\lambda), a(\lambda), b(\lambda)$, $b_{\mathrm{b}}(\lambda)$ ) considering the latter water classification.

Taking into account the aforementioned discussion, the corresponding attenuation of the optical signal can be described as [16]

$$
I=I_{0} \mathrm{e}^{-c(\lambda) d}
$$

Table II

VALUES FOR BEAM ATTENUATION COEFFICIENT, ABSORPTION COEFFICIENT, SCATTERING COEFFICIENT, BACKSCATTERED COEFFICIENT FROM [14]

\begin{tabular}{|c|c|c|c|c|}
\hline Water type & $c(\lambda)$ & $a(\lambda)$ & $b(\lambda)$ & $b_{\mathrm{b}}(\lambda)$ \\
\hline Pure seawater & 0.056 & 0.053 & 0.003 & 0.0006 \\
\hline Clear ocean & 0.150 & 0.069 & 0.080 & 0.0010 \\
\hline Coastal ocean & 0.305 & 0.088 & 0.216 & 0.0014 \\
\hline Turbid harbor & 2.170 & 0.295 & 1.875 & 0.0076 \\
\hline
\end{tabular}

in which $I$ is the light intensity at the receiver, $I_{0}$ is the light intensity at the transmitter, and $d$ is the distance between transmitter and receiver. One can define

$$
L(\lambda, d)=\mathrm{e}^{-c(\lambda) d}
$$

as the propagation loss factor illustrated in Figure 4(a) (with log-scale for the y-axis in Figure 4(b)).

\section{A. Noise in Underwater Optical Communications}

The main noise types impairing underwater optical transmissions are [2], [19]: excess noise, quantum shot noise, optical excess noise, optical background noise, photo-detector dark current noise, and electronic noise.

- Excess noise is generated in the process of amplifying the signal at the receiver, which is necessary for overcoming the effects of thermal noise;

- Quantum shot noise occurs due to random variations of the number of photons in the optical receiver [2];

- Optical excess noise is caused by transmitter imperfections;

- Optical background noise occurs due to environmental optical clutter;

- Photo-detector dark current noise is caused by electrical current leakage from photo-detector; 


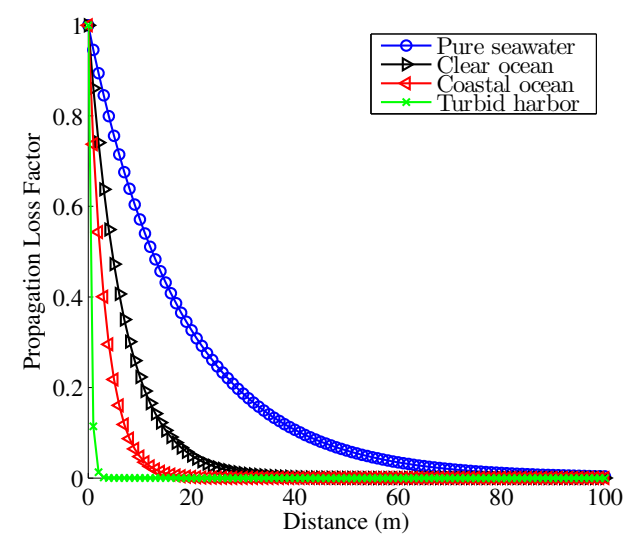

(a) Attenuation of the optical signal (propagation loss factor).

Figure 4. Channel properties for optical communications.

- Electronic noise comes from electronic components.

\section{B. Optical Transducers}

Transducers for underwater optical communications have different requirements depending on whether they are working as sensors at the receiver end or as actuators at the transmitter end. Transducers designed for generating optical signals from electrical signals are composed of optical source, projection optical system, and beam steering, whereas transducers for sensing optical signals and converting them into electrical signals are composed of collection optics and detector [2].

1) Transmitter: The optical source can be either a laser or a light-emitting diode (LED). In the case of laser sources, as each technology has distinct characteristics, the selection of the most appropriate one depends on the system requirements. One technology is argon-ion lasers, in which the electrical to optical conversion is extremely inefficient [2]. Other technologies include diode-pumped solid-state (DPSS) lasers, InGaN lasers, whose devices are much more expensive than LEDs [2] and are susceptible to over-current problems, and tunable lasers, which can adapt the frequency of emission in order to have a lower wave propagation attenuation according to the particular environmental characteristics. Another technology is the socalled laser modulators, whose data rates are extremely low (in the order of bps or kbps) and the propagation range is relatively longer (in the order of hundreds of meters) [2]. On the other hand, LEDs are cheaper optical sources when compared to lasers, but they have shorter propagation range [16].

The function of projection optics is to focus the beam toward a predefined direction. Beam steering is fundamental to the optical system performance. Indeed, transmitter and receiver have to establish a point-to-point spatially aligned connection so that the optical signals that arrive at the receiver end have enough energy to be reliably decoded. In this context, the concept of smart optical systems is emerging in which transmitters are able to estimate water quality through the backscattered signal in order to adapt the transmission power accordingly, thus improving the overall transmission process [16]. The smart transmitter might have an array of

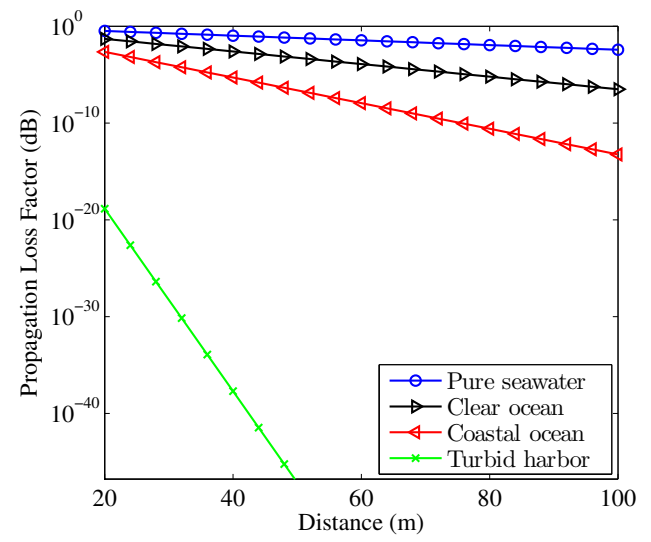

(b) Detail of the attenuation of the optical signal (propagation loss factor).
LEDs designed in hexagonal pyramid shape [16], where each LED has one lens. Each LED can be individually addressed for choosing an output direction, composing the switched beam steering mechanism at the transmitter side.

2) Receiver: Receivers are composed of collection optics and detector [2]. The collection optics can be a single lens, or an array of lenses, whose main role is to gather the transmitted rays. The detector is a photosensor, whose main role is to convert optical signal into electrical signal. The objective of the transducer at the reception end is to collect the maximum amount of photons that were transmitted. In order to improve system performance, some relevant characteristics of the collection optics and of the detector have to be analyzed and considered in the system design.

One characteristic is the aperture size of the photosensor. It is desirable to have a sensor with large aperture size. One photosensor with this characteristic is the photomultiplier tube (PMT). These sensors, however, can be expensive and bulky [16], which is a disadvantage for some applications. Another alternative for increasing the aperture size is to use an array of lenses in front of the small collection area photosensor.

An ideal photosensor should be cheap, small, robust, and power efficient [20], however, these requirements cannot be fulfilled simultaneously in the current technology stage. According to the system specifications, a particular type of photosensor must be chosen. The main photosensor types are [2], [13], [20]: photoresistors, photothyristors, phototransistors, photomultiplier tube (PMT), p-n photodiodes, avalanche photodiode (APD), photon detector selection, semiconductor photosensors, and biologically-inspired quantum photosensors (BQP).

\section{Main Issues in Optical Communications}

In optical communications it is important to quantify channel dispersion in order to configure the more appropriate signal-detection method for a reliable communication. The channel model is affected by features inherent to the physical layer, such as water type as well as effects related to the 
receiver lens' aperture size. Unlike other technologies, the optical transceiver requires special signal processing algorithms to track changes in the directionality of the receiver field of view and the optical transmitter beam. The possibility of channel interruption due to the passage of marine life should be detected on time to avoid loss of information. The optical transmitter can process the backscattered signal to determine the transmission power and/or the appropriate data rate. Signal processing also plays a key role by filtering the received signal to counter environmental noises that might entail time-domain, frequency-domain, and spacial filtering. The optical transceiver can be configured in closed loop where the receiver includes a diversity combiner and can inform the transmitter with power and data-rate controls obtained from the backscattering estimation. The receiver can also estimate the angle of arrival and inform the transmitter to keep the alignment as precise as possible.

Besides all aforementioned issues, the main drawback related to optical communications is their dependence on water turbidity. This environmental condition constrains the propagation distance, meaning that the propagation distance achievable when using optical technology may not be sufficient for some applications.

\section{Acoustic Communications}

As seen before, both RF and optical transmissions have limited propagation range. The former is severely affected by strong attenuation, which leads to a small propagation distance, whereas the latter depends on the water turbidity. Therefore, acoustic communication is an alternative technology to reach higher distances, currently being the dominant technology for wireless underwater communications.

The speed of propagation of waveforms depends on the electromagnetic or mechanical properties of the medium. Electromagnetic waves can propagate through air at speeds close to the speed of light in vacuum, which is around 4 to 5 orders of magnitude larger than the speed of propagation $c$ of acoustic waves in fluids. This imposes tremendous constraints on the overall transmission process using acoustic waves. Indeed, the parameters affecting the speed of propagation play a major role in acoustic-based communications. A model for the sound speed profile (SSP) for underwater environments with depth up to 1000 meters is [21] (in meters per second):

$$
\begin{aligned}
c=1449.2+4.6 T & -0.055 T^{2}+0.00029 T^{3} \\
& +(1.34-0.01 T)(S-35)+0.016 z,
\end{aligned}
$$

in which $T$ is the temperature (in degrees Celsius), $S$ is the salinity (in ppt - parts per thousand), and $z$ is the water depth (in meters). Another model for the SSP considering all depths can be found in [1]. It is worth mentioning that the propagation speed is always an increasing function of temperature, salinity, and depth. In case a more realistic model for the sound speed is needed, an in-situ CTD (conductivity, temperature, and depth) measurement must be performed in order to estimate the sound speed profile.

Signal propagation is another relevant issue in underwater acoustic communication. Multiple delayed and distorted

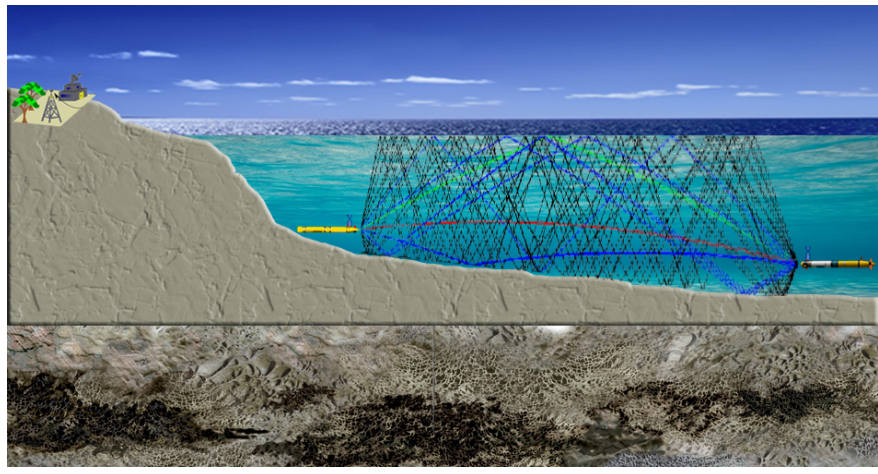

Figure 5. Example of a communication in shallow water environment. Multiple delayed and distorted versions of the transmitted signal arrives at the receiver-end.

versions of the transmitted signal arrive at the receiver due to the multipath channel, as shown in Figure 5. These phenomena generate distortions in the signal such as intersymbolinterference (ISI), that must be compensated by the transceiver. As a consequence, knowledge of the channel model might enable the design of more efficient transceivers [22]-[24], leading to communication with improved data rate. So, an actual concern is the characterization of the underwater acoustic channel [25]-[27], as well as its capacity [28], [29].

The acoustic waves propagate facing frequency-dependent attenuation and delay, and this fact plays a central role in the design of traditional wireless communication systems. Determining the attenuation behavior as a function of frequency is quite desirable for a system designer, since it gives technical support for the decision of frequency bands to be employed in the communication. The acoustic signal suffers little attenuation at low frequencies, and increasing attenuation at higher frequencies. Nonetheless, low frequency ranges and low speed of propagation are two major issues that might hinder high-throughput undersea communications. Indeed, low bandwidth imposes a constraint on the amount of bits that can be transmitted in each channel utilization, whereas the low speed of propagation increases the round-trip time and amplifies Doppler effect.

Taking into consideration the propagation properties, from a signal processing viewpoint, a given snapshot of the underwater channel could be characterized by its channel-impulse response. The channel transfer function might have nonminimum phase [30], thus implying that the inverse system is not stable. Such fact can turn the equalization process harder to implement. Some well-known equalization techniques applied for underwater acoustics are MMSE-based DFEs (minimum mean-squared error-based decision-feedback equalizers) [31], adaptive turbo equalizers [32], and TRM (time reversal mirror) [33], [34]. Another common problem occurs when the receiver is at a shadow zone, so that the received signals are relatively weak, causing loss of connection [30]. Other phenomena that yield variation in the sound propagation are tides, currents, and internal waves [35].

Furthermore, as for RF and optical technologies, it is common to classify acoustic communications considering the underwater environment. The underwater environment is clas- 
sified as shallow water and deep water, but each water type possesses two distinct definitions [36]: hypsometric and acoustic. In the hypsometric definition, shallow water is located in the continental shelf, in which the water column depth is mainly lower than 200 meters. Generally the sea bottom on the border of the continental shelf falls off rapidly into deep water, in which the water column has more than $2,000 \mathrm{~m}$ depth. The shallow water classification in acoustic definition considers that the acoustic waves reflect at the sea floor and at the sea surface before they are detected at the receiver, whereas in deep water, the wave does not necessarily reflect at the sea bottom.

Power loss in acoustic waves occurs due to three main phenomena: spreading loss, absorption loss, and scattering loss [30]. Spreading loss is the dispersion of a finite amount of energy that is transmitted by a source when the wave front is propagating over a large surface area. Depending on the distance, the wave surface is modeled either as a sphere or as a cylinder. For long ranges, spreading loss is modeled as cylindrical since the range of propagation is bounded by the sea floor and by the sea surface. Absorption loss, on the other hand, is the transformation of part of the transmitted energy of the acoustic wave into heat. High frequencies suffer large absorption losses. Similarly, longer propagation distances lead to higher absorption loss [37]. The third phenomenon is scattering, which is a modification of acoustic wave propagation due to obstacles. These obstacles can be caused by sea surface, sea floor, objects in the water, just to mention few examples. The aforementioned spreading loss and absorption loss phenomena contribute to path loss, whose simplified model is expressed in $\mathrm{dB}$ as [4], [37]-[39]:

$$
\begin{aligned}
10 \log A(l, f)=\underbrace{10 \log A_{0}}_{\mathrm{NF}} & +10 k \log l \\
& +l \underbrace{10 \log a(f, S, T, c, p H, z)}_{\alpha(f, S, T, c, p H, z)}
\end{aligned}
$$

where $l$ is the distance (in meters) between transmitter and receiver, $f$ is the frequency (in $\mathrm{kHz}$ ), $k$ is the spreading factor, whose commonly employed values are: 1 for cylindrical spreading, 2 for spherical spreading, and 1.5 for "practical spreading" [37]. Parameter NF $=10 \log A_{0}$ is a normalization factor that can be related to the inverse of the transmitted power. Variable $\alpha(f, S, T, c, p H, z)$ represents the attenuation coefficient (in $\mathrm{dB} / \mathrm{m}$ ) that depends on environmental conditions [39]: frequency ( $f$ in $\mathrm{kHz})$, salinity $(S$ in ppt), temperature ( $T$ in degree Celsius), speed of propagation of the acoustic wave $(c$ in $\mathrm{m} / \mathrm{s}), \mathrm{pH}$, and water depth $(z$ in $\mathrm{m})$. Typically, for shallow water, spreading is considered to be cylindrical, whereas for deep water, spreading is considered to be spherical at positions relatively near to the transmitter.

A numerical example of the dependence of path loss on several factors, such as frequency and distance between transmitter and receiver, is depicted in Figures 6(a) and 6(b) for shallow and deep waters, respectively. For computing path loss, the factor NF was considered as zero, $k=1$ for shallow water, and $k=2$ for deep water. For a fixed distance, there is a minimum loss value that is related to the spreading loss and the attenuation factor $(l \alpha(f, S, T, c, p H, z))$ dominates in higher frequencies. From these figures, it is possible to observe that, for low frequencies, the path loss is approximately the same for different distances. Path loss increases considerably when the frequency and the distance between transmitter and receiver increases.

A possible multipath model for the acoustic environment has the channel coefficients inversely proportional to the path loss, and to other additional transmission losses, such as reflection and scattering losses.

\section{A. Noise in Underwater Acoustic Communications}

Noise in acoustic communications can be classified as ambient noise, or as external interference [40]. There are many sources of ambient noise in deep water and the influence of each source occurs at specific frequency ranges [37]: for $f<10 \mathrm{~Hz}$, earthquakes, turbulence in the ocean and in the atmosphere, distant storms, and underwater vulcan eruptions are the main sources of noise; for $10<f<100 \mathrm{~Hz}$, traffic of distant shipping can be an issue; for $100 \mathrm{~Hz}<f<100 \mathrm{kHz}$, the state of the sea surface and of the wind speed are important factors; and for $f>100 \mathrm{kHz}$, thermal noise is the main issue. The noise power spectrum density of the ambient noise decreases as frequency increases (up to $f=100 \mathrm{kHz}$ ) and may vary according to water depth, ocean location, and time [41].

External interference includes marine animals (biological noise), ice cracking, among others. They produce acoustic waves whose frequencies are in a sub-range of $100 \mathrm{~Hz}$ $<f<200 \mathrm{kHz}$, causing interference in the received signal. These noise properties depend on the ocean area and might be intermittent [42].

\section{B. Acoustic Transducers}

Acoustic transducers convert electrical signals into sound (transmitter) or sound into electrical signals (receiver). Transmitters are called sources or projectors, and receivers are called hydrophones. However, commonly a single transducer acts as transmitter and receiver in acoustic modems. These devices are designed for underwater environments and can be attached to floating objects (e.g. boat or buoy) or can be moored.

Generally sources or projectors work in particular frequency bands, that are generally narrower than the hydrophone frequency band. Projectors can be omnidirectional or hemispherical, whereas hydrophones can be omnidirectional or directional. Several omnidirectional hydrophones can compose an array, so that when the acquired signals are properly combined, it is possible to select a preferential receiving direction, or to use diversity for improving system performance.

The most common types of transducers are piezoelectric and magnetostrictive [21]. Other less common types include parametric or finite-amplitude sources and receivers.

\section{Main Issues in Underwater Acoustic Communications}

Most acoustic communication links demand mitigation of the Doppler effect, that might utilize fast learning and tracking adaptive algorithms such as Kalman filters. The dispersive 


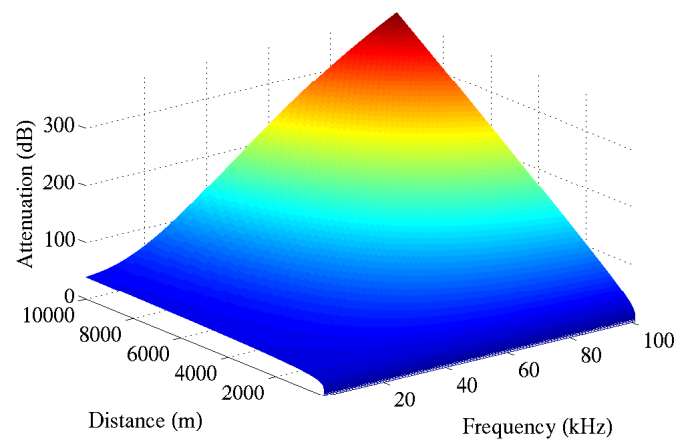

(a) Shallow water - ocean depth $60 \mathrm{~m}$.

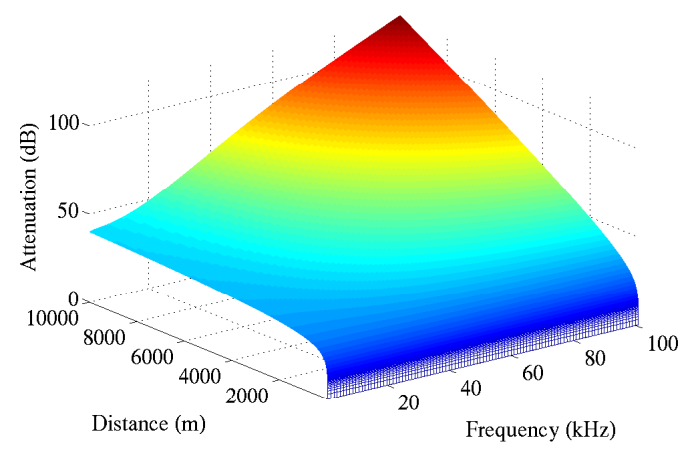

(b) Deep water - ocean depth $10 \mathrm{~km}$.

Figure 6. Channel property for acoustic communications.

characteristics of the acoustic channel model is another important issue to guarantee successful equalization and synchronization. The use of multicarrier transceivers is also possible as a solution to channel estimation but the high Doppler effect in acoustic communication affects subchannel orthogonality of the subcarriers. The propagation delay of the acoustic signal places additional constraints on the signal processing solution utilized in these systems; a typical example is the challenge of deploying relay sensor networks.

The relative velocity between transmitter and receiver ends, and the underwater dynamical environment cause the Doppler phenomenon. Although also present in radio-frequency communication, Doppler effect is particularly noticeable when communicating using pressure waves, due to the relatively low speed of propagation of the wavefront. The effect is even more pronounced for underwater acoustic communication, since in this case, buoys and vessels which host transmitter and receiver are seldom at absolute rest. Therefore, Doppler distortion is hard to ignore, and mitigating its effect is of paramount importance for an efficient communication system.

Doppler effect produces time warping on the transmitted signal. In addition to the expansion or contraction of the signal duration, time warping also distorts signal phase. Time warping can be seen as a modification of the time index from $t$ to $\bar{t}=[t+\rho(t)]$, where $\rho(t)$ can be approximated by $\left(v_{R} \cos \phi+v_{T} \cos \theta\right) / c$, in which $v_{R}, v_{T}$ are the receiver and the transmitter speeds, respectively, and the angles $\phi$ and $\theta$ are shown in Figure 7. The reader should keep in mind that speeds and angles are expected to vary with time, therefore $\rho(t)$ holds explicitly its time dependence. Once the Doppler factor $\rho(t)$ is estimated, this effect may be compensated by proper adjustment of the sampling frequency, followed by a signal phase correction [43]. A residual distortion may remain, which is usually treated in the channel estimation procedure. Therefore, any Doppler estimation scheme implemented must be robust and resilient. In order to address this issue, new approaches for estimating and compensating Doppler effect have been studied in [44]-[57]. Nonetheless, there is no consensus about the optimal way to tackle this problem.

Another concerning issue in underwater acoustic communications is related to the channel impulse response. As the

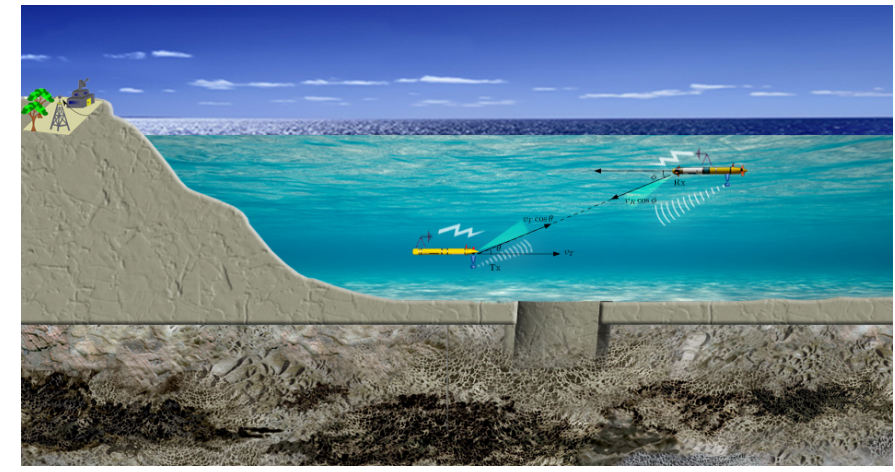

Figure 7. Transmitter and receiver moving with respect to the propagation medium.

speed of propagation of acoustic waves is lower than the speed of propagation of electromagnetic waves in the air, the coherence time of the acoustic channel is smaller than that for the aerial RF channel. Indeed, a channel possessing a higher delay spread might require a receiver with higher computational complexity, due to the augmented number of variables. A possible approach to avoid this complexity increment is to employ the knowledge that the acoustic channel is intrinsically sparse. For references which exploit this property for estimating the channel impulse response, see [47], [58][63].

Channel equalization is another big issue in underwater acoustic communication. Those channel features mentioned above may introduce severe intersymbol-interference (ISI) and intercarrier interference (ICI) in the received signal. The equalizer should be capable of removing and compensating these distortions. Some techniques, such as MMSE-based DFEs (minimum mean-squared error-based decision-feedback equalizers) [31] and TRM (time reversal mirror) [33], [34], [64], have been reported in the literature. Nonetheless, equalization and channel decoding could be implemented in an iterative way, exchanging pieces of information that help to achieve a better performance. Turbo equalizers are instances of those systems that implement such iterative process and that have been extensively employed and investigated [32], [65][68]. 
As discussed in Sections III, IV, and V, each technology has its constraints, which can limit the communication range, throughput, or even hinder a reliable communication. One way to overcome this hindrance is through the usage of underwater networks.

\section{UNDERWATER NETWORKS}

Underwater networks (UWNs) are useful in several different scenarios that rely on data retrieval, exchange of control commands among pieces of equipment, or in a broader sense, general information transfer. Examples of these scenarios appear in seismology, oceanography, marine life monitoring and surveillance, natural disaster prevention and control, integrity check of oil \& gas facilities, and military tactical operations. The network requirements of these applications differ very much and also depend upon the particular transmission technology which is employed by the network nodes. The network nodes must have a multitude of functionalities, including measuring, storing, and transmitting capabilities using possibly distinct transmission technologies.

For example, it is common that oil \& gas industrial applications of UWNs employ a set of network nodes fixed at the ocean bottom that have to exchange information with other nodes on the ocean surface or with an onshore node. In order to do that, the nodes at the ocean bottom may have to use multi-hop communication in order to transfer their information through an underwater gateway node, which is able to perform all necessary protocol conversions to other types of networks. Furthermore, even the nodes at the ocean bottom may have to communicate with each other in order to estimate some parameter of interest in a cooperative way so that a decision could be taken in an efficient and fast way. This type of application needs devices in the underwater environment which are somehow (logically) connected to each other through a UWN.

As described before, the particular example in which one has to send or receive data to or from a submarine with unknown position may require covering a vast area encompassing several square kilometers. On the other hand, retrieving information from a monitoring device in oil wells could be performed by an ROV (remotely operated vehicle) in short range using low power. In addition, ROVs and AUVs pose new challenges to UWNs since they can act like nodes possibly requiring a large bandwidth for video and audio content transfer. All these examples make clear that the general requirements for deploying UWNs are quite difficult to list, since the specific application can impose rather different constraints. Indeed, the coexistence of wireless and wired UWNs seems to be a natural solution in order to fulfill the system requirements.

\section{A. UWN Requirements}

Nodes should be capable of storing a significant amount of data. Indeed, there are many applications in which some of the nodes in the underwater environment are constantly receiving a large amount of data acquired using different types of sensors that are monitoring physical variables, such as pressure, temperature, and flow. Hence, UWN nodes may require large buffers for storing data before transmitting them.

Due to the aforementioned massive amount of data, another general requirement associated with UWNs is pursuing a protocol stack that allows efficient transmissions. In other words, one cannot spend much bandwidth with headers and redundant information. In addition, data compression should be employed whenever possible, and the physical layer has to play its role by working as closely as possible to the particular channel capacity [28], [29].

Usually, the energy required to transmit signals in the underwater environment, especially mechanical waves employed by acoustic technology, is much larger than the energy required to listen, receive, and process the received data. Since batteries are not easily rechargeable or replaceable in some UWN nodes, the architecture of UWNs and even the network protocols must be devised taking this fact into account. Indeed, low power consumption to avoid frequent trips to recharge nodes is highly desirable. Energy efficiency is a key issue in UWNs.

Another UWN requirement is that the sturdy nodes must be capable of sustaining impacts during and after installation, in addition to being robust so that they can operate in unfriendly circumstances. Besides, flexible nodes are also important to accommodate different needs, different configurations, and different interfaces.

Also, the protocol stack of UWNs must guarantee the reliability of the overall transmission. One must remember that the underwater environment is a challenging communication channel, so that guaranteeing reliability of the transmission/reception process is not an easy task, especially when energy efficiency and high data rates are also desirable targets.

As stated before, acoustic signals traveling the underwater environment usually have much smaller speed of propagation as compared to RF signals propagating through the air. This means that the delay associated with packet exchanges among UWN nodes can be very large. Besides, as the acoustic links are subjected to link outages, UWN protocols must be delaytolerant.

As the underwater channel imposes so many harsh constraints on the transmission, the routing of packets between UWN nodes should rely on some sort of channel awareness. This means that cross-layer designs can play a key role in the context of UWN protocols.

\section{B. Main Issues in UWNs}

Besides all aforementioned issues, it is worth describing how the PHY-layer affects UWNs with respect to their architecture, design, and operation. Let us start by first mentioning that pure optical-based functional UWNs are still lacking at the time of writing this paper since there exists some restriction when using currently available technology: almost all UWN nodes would only be able to work with point-topoint communication in which the nodes must be correctly aligned, thus constraining the network architecture. The reader should keep in mind that the UWN nodes may be mobile and that even those anchored nodes cannot be taken as completely 
fixed due to natural movements under water. This is a possible explanation for having so few works addressing UWNs using only optical transmissions [16], [69].

Regarding pure RF-based functional UWNs, one has the well-known problem of short-range communication associated with RF signals under water, especially seawater. This also imposes a huge constraint on the UWN architecture, since it would not be possible to have nodes placed too far from each other. A partial solution to this problem is to work with RF signals with extremely low frequencies, so that the range of communication is larger. However, this solution works against achieving high transmission data rates due to the consequent bandwidth reduction, in addition to the problems related to antenna size. In addition, at low frequencies the speed of propagation of the electromagnetic wave is lower [7], thus increasing the delay-tolerance constraints of the corresponding UWN protocols. That is why it is hard to find works describing fully functional UWNs using only RF transmissions. A discussion about some aspects of RF-based UWNs can be found in [7], [70].

An overwhelming majority of works describing solutions for UWNs rely on acoustic transmissions (see, for example, [3], [4], [71]-[80] and references therein). Acoustic technology allows larger distances between UWN nodes. In addition, there is no need for aligning the nodes, which thus simplifies UWN operation and architecture design. However, the amount of energy (or the energy efficiency in bits per Joule) spent by the projectors to generate the acoustic mechanical waves can be much larger than the energy associated with receiving, processing, and storing the information. Indeed, this is related to generating mechanical waves that can travel through the water over kilometers, for example. Thus, UWN protocols must be aware of this fact.

A possible solution to this issue is data fragmentation and the correct choice of the length of the signal frame. The authors in [71], for example, propose an optimization problem for defining the optimal length for the transmitted signals, taking into account bit-error probability, which directly affects the average number of retransmissions (most of UWN applications require reliability), the header and raw data lengths, and the consumption related to each individual step of the transmission/reception process, including the energy spent in control channels. Moreover, UWN architecture and operation must take into account that it may be better to relay the information using multi-hops than spending a huge amount of energy trying to reach far away nodes. All of these facts must be considered in efficient designs of UWNs, thus showing how different a protocol stack can be from commonly used protocols for RF transmissions in the air.

The propagation speed of acoustic waves is relatively small, which means that large propagation delays are inherent to UWNs. In addition, UWNs also have to deal with link outages. Therefore, the protocol must be able to handle these two features, which is not the case of the traditional TCP/IP protocol.

We believe that an efficiently designed protocol stack for UWNs must consider using all types of transmission technologies, namely optical, RF, and acoustic [81]. The UWN nodes should be self-reconfigurable, depending on the transmission data-rate demands, neighboring nodes which they can communicate with using a particular type of technology, and target energy efficiency [82].

\section{RF, OpticAl or Acoustic TeChNOLOgy?}

Before concluding which technology should be the best to transport information, one must know in which environmental conditions the system has to operate, as well as what are the communication requirements. Table III summarizes the main features and drawbacks inherent to each technology that might help approach a proper solution. This table compares the water properties that mostly affect each transmission technique: salinity for RF, water turbidity for optical, and water depth for acoustic. Each technology is mainly affected by distinct water features, as described in Table III. The interested reader can refer to [83]-[90] for further information regarding achievable data rates for different technologies and parameters.

In addition, to achieve robust and reliable underwater communication, the challenge is to propose flexible communication systems including all the aforementioned communication technologies. This flexible system could be intelligent so that the maximum transmission rate could be achieved considering, for instance, environmental conditions, distance, and relative movement between transmitter and receiver. This heterogeneous system would be able to switch technology of transmission/reception according to a predefined cost function: the receiver would send from time to time an acknowledgment signal to the transmitter that would take the appropriate action. Furthermore, since all underwater communication systems have inherent limitations with respect to connections over long distances, the use of networks including several sensors and relays, with the aid of smart protocols, seems to be a natural solution. The network nodes could be fixed or mobile, and all nodes should ideally be able to transmit and/or receive with the three technologies [90]. The mobile nodes should be smart enough such that moving to a nearby position would result in improved communication by optimizing an appropriate cost function.

\section{CONCLUding Remarks}

Proper exploitation of the ocean environment for communications requires a clear understanding of the mechanisms affecting the underwater signal, such as the attenuation characteristics originated from the propagation properties of RF, optical, and acoustic transmissions. Modeling underwater signal propagation is very difficult but its understanding plays a key role to determine the effective data processing at the transmitter and at the receiver so that reliable and accurate communications are possible. As expected, each communication technology requires distinct channel modeling, turning the task of conceiving a network employing flexible modems much more challenging.

Future generation modems for certain will include many signal processing tools in order to achieve high-data rates at the physical layer when employing any of the technologies 
available or a combination of them whenever the environmental conditions allow. Reaching data rates nearing theoretical channel(s) capacity(ies) is a desired objective to be accomplished with the indispensable aid of today's ubiquitous signal processing tools. This paper contributes in this direction by providing an up-to-date survey of the main technical aspects and research challenges of wireless underwater communications.

\section{ACKNOWLEDGMENT}

We would like to thank the reviewers for their valuable and very constructive comments.

\section{REFERENCES}

[1] X. Lurton, An Introduction to Underwater Acoustics: Principles and Applications, 2nd ed. Springer, 2010.

[2] M. Lanzagorta, Underwater Communication, ser. Synthesis Lectures on Communications. Morgan \& Claypool Publishers, 2012, vol. 5.

[3] I. F. Akyildiz, D. Pompili, and T. Melodia, "Underwater acoustic sensor networks: research challenges ," Ad Hoc Networks, vol. 3, no. 3, pp. 257-279, May 2005. doi: http://dx.doi.org/10.1016/j.adhoc.2005.01.004

[4] T. Melodia, H. Kulhandjian et al., Advances in Underwater Acoustic Networking, 1st ed. Wiley, 2012, ch. 23.

[5] Y. Chen, W. yan Pan et al., "The ELF/VLF field at the depth of submarine excited by satellite electropult," in Antennas Propagation and EM Theory (ISAPE), 2010 9th International Symposium on, 2010. doi: 10.1109/ISAPE.2010.5696513 pp. 505-508.

[6] R. Dowden, R. Holzworth et al., "World-wide lightning location using vlf propagation in the earth-ionosphere waveguide," Antennas and Propagation Magazine, IEEE, vol. 50, no. 5, pp. 40-60, 2008. doi: 10.1109/MAP.2008.4674710

[7] X. Che, I. Wells et al., "Re-Evaluation of RF Electromagnetic Communication in Underwater Sensor Networks," Communications Magazine, IEEE, vol. 48, no. 12, pp. 143-151, 2010. doi: 10.1109/MCOM.2010.5673085

[8] A. Zoksimovski, D. Sexton et al., "Underwater electromagnetic communications using conduction channel characterization," in 7th ACM International Conference on Underwater Networks \& Systems, Los Angeles, CA, USA, November 2012.

[9] D. Sexton, "Subsea Communications, RPSEA Final Report," GE Global Research, Tech. Rep., December 2011.

[10] F. Hanson and S. Radic, "High bandwidth underwater optical communication," Appl. Opt., vol. 47, no. 2, pp. 277-283, Jan 2008. doi: 10.1364/AO.47.000277

[11] D. Anguita, D. Brizzolara, and G. Parodi, Prospects and Problems of Optical Diffuse Wireless Communication for Underwater Wireless Sensor Networks, Wireless Sensor Networks: Application - Centric Design. InTech, 2010, ch. 12.

[12] S. Tang, Y. Dong, and X. Zhang, "On link misalignment for underwater wireless optical communications," Communications Letters, IEEE, vol. 16, no. 10, pp. 1688-1690, August 2012. doi: 10.1109/LCOMM.2012.081612.121225

[13] J. Simpson, "A 1 Mbps Underwater Communications System using LEDs and Photodiodes with Signal Processing Capability," Master's thesis, North Carolina State University, Raleigh, North Carolina, USA, 2007.

[14] C. Gabriel, M. Khalighi et al., "Monte-Carlo-Based Channel Characterization for Underwater Optical Communication Systems," $J$. Opt. Commun. Netw., vol. 5, no. 1, pp. 1-12, January 2013. doi: 10.1364/JOCN.5.000001

[15] V. I. Haltrin, "Chlorophyll-based model of seawater optical properties," Appl. Opt., vol. 38, no. 33, pp. 6826-6832, Nov 1999. doi: 10.1364/AO.38.006826

[16] J. Simpson, B. Hughes, and J. Muth, "Smart Transmitters and Receivers for Underwater Free-Space Optical Communication," Selected Areas in Communications, IEEE Journal on, vol. 30, no. 5, pp. 964-974, June 2012. doi: 10.1109/JSAC.2012.120611

[17] S. Arnon, "An underwater optical wireless communication network," in Proc. SPIE 7464, Free-Space Laser Communications IX, 2010.
[18] B. Cochenour, L. Mullen, and A. Laux, "Characterization of the BeamSpread Function for Underwater Wireless Optical Communications Links," Oceanic Engineering, IEEE Journal of, vol. 33, no. 4, pp. 513521, 2008. doi: 10.1109/JOE.2008.2005341

[19] S. Jaruwatanadilok, "Underwater wireless optical communication channel modeling and performance evaluation using vector radiative transfer theory," Selected Areas in Communications, IEEE Journal on, vol. 26, no. 9, pp. 1620-1627, 2008. doi: 10.1109/JSAC.2008.081202

[20] H. Brundage, "Designing a wireless underwater optical communication system," Master's thesis, Massachusetts Institute of Technology, Cambridge, MA, USA, 2010.

[21] F. B. Jensen, W. A. Kuperman et al., Computational Ocean Acoustics, 2nd ed., ser. Modern Acoustics and Signal Processing. Springer Publishers, 2011.

[22] M. Stojanovic, "Recent advances in high-speed underwater acoustic communications," IEEE Journal of Oceanic Engineering, vol. 21, no. 2, pp. 125-136, Apr 1996. doi: 10.1109/48.486787

[23] D. B. Kilfoyle and A. B. Baggeroer, "The state of the art in underwater acoustic telemetry," IEEE Journal of Oceanic Engineering, vol. 25, no. 1, pp. 4-27, Jan 2000. doi: 10.1109/48.820733

[24] M. Chitre, S. Shahabudeen, and M. Stojanovic, "Underwater acoustic communications and networking: Recent advances and future challenges," Marine Technology Society Journal, vol. 42, no. 1, pp. 103-116, 2008. doi: https://doi.org/10.4031/002533208786861263

[25] P. Qarabaqi and M. Stojanovic, "Statistical modeling of a shallow water acoustic communication channel," in Proc. Underwater Acoust. Meas. Conf., Nafplion, Greece, June 2009, pp. 1341-1350.

[26] _ "Statistical characterization and computationally efficient modeling of a class of underwater acoustic communication channels," Oceanic Engineering, IEEE Journal of, vol. 38, no. 4, pp. 701-717, Oct 2013. doi: 10.1109/JOE.2013.2278787

[27] M. Chitre, "A high-frequency warm shallow water acoustic communications channel model and measurements," The Journal of the Acoustical Society of America, vol. 122, no. 5, pp. 2580-2586, 2007. doi: http://dx.doi.org/10.1121/1.2782884

[28] J. M. Passerieux, F. X. Socheleau, and C. Laot, "On the capacity of the underwater acoustic communication channel under realistic assumptions," in Wireless Conference 2011 - Sustainable Wireless Technologies (European Wireless), 11th European, April 2011, pp. 1-6.

[29] _ - "Achievable rates over doubly selective rician-fading channels under peak-power constraint," IEEE Transactions on Wireless Communications, vol. 12, no. 2, pp. 586-594, February 2013. doi: 10.1109/TWC.2012.122212.111812

[30] J. Preisig, "Acoustic Propagation Considerations for Underwater Acoustic Communications Network Development," SIGMOBILE Mobile Computing and Communications Review, vol. 11, no. 4, pp. 2-10, October 2007. doi: $10.1145 / 1347364.1347370$

[31] A. B. Baggeroer, "An overview of acoustic communications from 2000-2012," in Underwater Communications: Channel Modelling \& Validation (UComms). Sestri Levante, Italy: NATO Centre for Maritime Research and Experimentation, September 2012.

[32] J. W. Choi, T. Riedl et al., "Adaptive Linear Turbo Equalization Over Doubly Selective Channels," Oceanic Engineering, IEEE Journal of, vol. 36, no. 4, pp. 473-489, 2011. doi: 10.1109/JOE.2011.2158013

[33] H. C. Song, "An overview of underwater time-reversal communication," IEEE Journal of Oceanic Engineering, vol. PP, no. 99, pp. 1-12, 2015. doi: 10.1109/JOE.2015.2461712

[34] M. Fink, "Time-Reversed Acoustics," Scientific American, pp. 91-97, November 1999.

[35] P. van Walree, "Propagation effects in underwater acoustic communication channels," in Underwater Communications: Channel Modelling \& Validation (UComms). Sestri Levante, Italy: NATO Centre for Maritime Research and Experimentation, September 2012.

[36] P. C. Etter, Underwater Acoustic Modeling and Simulation, 3rd ed. Spon Press, 2003.

[37] M. Stojanovic, "On the Relationship Between Capacity and Distance in an Underwater Acoustic Communication Channel,' SIGMOBILE Mobile Computing and Communications Review, vol. 11, no. 4, pp. 34-43, October 2007. doi: 10.1145/1347364.1347373

[38] M. Stojanovic and J. Preisig, "Underwater acoustic communication channels: Propagation models and statistical characterization," Сотmunications Magazine, IEEE, vol. 47, no. 1, pp. 84-89, January 2009. doi: 10.1109/MCOM.2009.4752682

[39] M. C. Domingo, "Overview of Channel Models for Underwater Wireless Communication Networks ," Physical Communication, vol. 1, no. 3, pp. 163-182, 2008. doi: http://dx.doi.org/10.1016/j.phycom.2008.09.001 
Table III

WIRELESS UNDERWATER TECHNOLOGIES: RF, OPTICAL AND ACOUSTIC

\begin{tabular}{|c|c|c|c|}
\hline & \multicolumn{3}{|c|}{ Technology } \\
\hline Main issues & RF & Optical & Acoustics \\
\hline $\begin{array}{l}\text { Key water } \\
\text { property }\end{array}$ & Salinity & Water turbidity & Water depth \\
\hline Water types & Fresh water $\times$ Seawater & $\begin{array}{c}\text { Main Jerlov water types: } \\
\text { Clearest water, Intermediate water, } \\
\text { Murkiest water } \\
\text { - From [14], [18]: Pure seawater, } \\
\text { Clear ocean water, Coastal ocean } \\
\text { water, Turbid harbor and estuary } \\
\text { water } \\
\end{array}$ & Shallow water $\times$ Deep water \\
\hline $\begin{array}{l}\text { Drawbacks } \\
\text { and/or } \\
\text { requirements }\end{array}$ & $\begin{array}{l}\text { High attenuation over short } \\
\text { distances }\end{array}$ & $\begin{array}{l}\text { - Line of sight link } \\
\text { - Receiver direction tracking } \\
\text { - Subject to marine fouling }\end{array}$ & $\begin{array}{l}\text { - Doppler estimation and } \\
\text { compensation } \\
\text { - Latency in communication } \\
\text { - Existence of shadow zones }\end{array}$ \\
\hline $\begin{array}{c}\text { Main } \\
\text { characteristics }\end{array}$ & $\begin{array}{c}\text { Can cross water/air surface } \\
\text { (boundary) }\end{array}$ & Achieve higher data rates & Propagates over longer distances \\
\hline $\begin{array}{l}\text { Reliable } \\
\text { communication } \\
\text { distance }\end{array}$ & Few meters & Tens of meters & Kilometers \\
\hline $\begin{array}{l}\text { Achievable data } \\
\text { rates }\end{array}$ & $\begin{array}{l}1 \text { to } 10 \text { Mbps (@1 - } 2 \text { m, [91]) } \\
50 \text { to } 100 \text { bps (@200 m, [91]) }\end{array}$ & $\begin{array}{l}1 \text { Gbps (@2 m, [10]) } \\
1 \text { Mbps (@25 m, [89]) }\end{array}$ & $\begin{array}{c}1.5 \text { to } 50 \mathrm{kbps}(@ 0.5 \mathrm{~km},[92]) \\
0.6 \text { to } 3.0 \mathrm{kbps} \\
\text { (@28 - } 120 \mathrm{~km},[93])\end{array}$ \\
\hline $\begin{array}{l}\text { Dependence of } \\
\text { the speed } \\
\text { propagation }\end{array}$ & $\begin{array}{l}\text { Frequency, water conductivity } \\
\text { (salinity and temperature) }\end{array}$ & $\begin{array}{c}\text { Frequency, water turbidity } \\
\text { (chlorophyll concentration, salt } \\
\text { ions, etc.) }\end{array}$ & Temperature, salinity, water depth \\
\hline
\end{tabular}

[40] S. Zhou and Z. Wang, OFDM for Underwater Acoustic Communication, 1st ed. Wiley, 2014.

[41] R. J. Urick, Principles of Underwater Sound, 3rd ed. McGraw-Hill, 1996.

[42] L. M. Brekhovskikh and Y. P. Lysanov, Fundamentals of Ocean Acoustics, 3rd ed., ser. Modern Acoustics and Signal Processing. Springer Publishers, 2003.

[43] T. Riedl, A. Bean, and A. Singer, "Broadband Doppler Compensation: Experimental Evaluation," in Underwater Communications: Channel Modelling \& Validation (UComms). Sestri Levante, Italy: NATO Centre for Maritime Research and Experimentation, September 2012.

[44] T. Riedl and A. Singer, "Broadband Doppler Compensation: Principles and New Results," in Signals, Systems and Computers (ASILOMAR), 2011 Conference Record of the Forty Fifth Asilomar Conference on, 2011. doi: 10.1109/ACSSC.2011.6190149 pp. 944-946.

[45] R. Diamant, A. Feuer, and L. Lampe, "Choosing the Right Signal: Doppler Shift Estimation for Underwater Acoustic Signals," in 7th ACM International Conference on Underwater Networks \& Systems, Los Angeles, CA, USA, November 2012.

[46] K. Perrine, K. Nieman et al., "Doppler estimation and correction for shallow underwater acoustic communications," in Signals, Systems and Computers (ASILOMAR), 2010 Conference Record of the Forty Fourth Asilomar Conference on, 2010. doi: 10.1109/ACSSC.2010.5757663 pp. 746-750.

[47] O. Rabaste and T. Chonavel, "Estimation of multipath channels with long impulse response at low snr via an mcmc method," Signal Processing, IEEE Transactions on, vol. 55, no. 4, pp. 1312-1325, 2007. doi: 10.1109/TSP.2006.888060

[48] C. H. Yuen and B. Farhang-Boroujeny, "Doppler scaling correction in ofdm," in Communications (ICC), 2013 IEEE International Conference on, June 2013. doi: 10.1109/ICC.2013.6655317 pp. 4713-4717.

[49] A. Abdelkareem, B. Sharif et al., "Low-complexity doppler compensation for ofdm-based underwater acoustic communication systems," in OCEANS, 2011 IEEE - Spain, June 2011. doi: 10.1109/OceansSpain.2011.6003384 pp. 1-6.

[50] T. Kang and R. Iltis, "Fast-varying doppler compensation for underwater acoustic ofdm systems," in Signals, Systems and Computers,
2008 42nd Asilomar Conference on, October 2008. doi: 10.1109/ACSSC.2008.5074548 pp. 933-937.

[51] A. Kibangou, L. Ros, and C. Siclet, "Doppler estimation and data detection for underwater acoustic zf-ofdm receiver,' in Wireless Communication Systems (ISWCS), 2010 7th International Symposium on, September 2010. doi: 10.1109/ISWCS.2010.5624286 pp. 591-595.

[52] B. Li, S. Zhou et al., "Multicarrier communication over underwater acoustic channels with nonuniform doppler shifts," Oceanic Engineering, IEEE Journal of, vol. 33, no. 2, pp. 198-209, April 2008. doi: 10.1109/JOE.2008.920471

[53] S. Mason, C. Berger et al., "Detection, synchronization, and doppler scale estimation with multicarrier waveforms in underwater acoustic communication," Selected Areas in Communications, IEEE Journal on, vol. 26, no. 9, pp. 1638-1649, December 2008. doi: 10.1109/JSAC.2008.081204

[54] J. Trubuil and T. Chonavel, "Accurate doppler estimation for underwater acoustic communications," in OCEANS, 2012 - Yeosu, May 2012. doi: 10.1109/OCEANS-Yeosu.2012.6263500 pp. 1-5.

[55] K. Tu, T. Duman et al., "Multiple-resampling receiver design for ofdm over doppler-distorted underwater acoustic channels," Oceanic Engineering, IEEE Journal of, vol. 38, no. 2, pp. 333-346, April 2013. doi: 10.1109/JOE.2012.2221812

[56] Y. Zakharov and V. Kodanev, "Multipath-doppler diversity of ofdm signals in an underwater acoustic channel," in Acoustics, Speech, and Signal Processing, 2000. ICASSP '00. Proceedings. 2000 IEEE International Conference on, vol. 5, 2000. doi: 10.1109/ICASSP.2000.861150 pp. 2941-2944.

[57] C. H. Yuen and B. Farhang-Boroujeny, "Non-linear doppler scaling correction in underwater acoustic channels: Analysis and simulation," in Oceans - San Diego, 2013, Sept 2013, pp. 1-7.

[58] W. Li and J. C. Preisig, "Estimation of rapidly time-varying sparse channels," IEEE Journal of Oceanic Engineering, vol. 32, no. 4, pp. 927-939, Oct 2007. doi: 10.1109/JOE.2007.906409

[59] M. Stojanovic, "Ofdm for underwater acoustic communications: Adaptive synchronization and sparse channel estimation," in 2008 IEEE International Conference on Acoustics, Speech and Signal Processing, March 2008. doi: 10.1109/ICASSP.2008.4518853 pp. 5288-5291. 
[60] C. R. Berger, S. Zhou et al., "Sparse channel estimation for multicarrier underwater acoustic communication: From subspace methods to compressed sensing," IEEE Transactions on Signal Processing, vol. 58, no. 3, pp. 1708-1721, March 2010. doi: 10.1109/TSP.2009.2038424

[61] T. Kang and R. A. Iltis, "Iterative carrier frequency offset and channel estimation for underwater acoustic ofdm systems," IEEE Journal on Selected Areas in Communications, vol. 26, no. 9, pp. 1650-1661, December 2008. doi: 10.1109/JSAC.2008.081205

[62] K. Pelekanakis and M. Chitre, "Adaptive sparse channel estimation under symmetric alpha-stable noise," IEEE Transactions on Wireless Communications, vol. 13, no. 6, pp. 3183-3195, June 2014. doi: 10.1109/TWC.2014.042314.131432

[63] J. Li and Y. V. Zakharov, "Sliding window adaptive filter with diagonal loading for estimation of sparse uwa channels," in OCEANS 2016 Shanghai, April 2016. doi: 10.1109/OCEANSAP.2016.7485346 pp. 15 .

[64] M. Fink, "Time Reversal of Ultrasonic Fields - Part I: Basic Principles," Ultrasonics, Ferroelectrics and Frequency Control, IEEE Transactions on, vol. 39, no. 5, pp. 555-566, September 1992. doi: 10.1109/58.156174

[65] M. Tuchler, R. Koetter, and A. C. Singer, "Turbo equalization: principles and new results," IEEE Transactions on Communications, vol. 50, no. 5, pp. 754-767, May 2002. doi: 10.1109/TCOMM.2002.1006557

[66] R. Otnes and M. Tuchler, "Iterative channel estimation for turbo equalization of time-varying frequency-selective channels," IEEE Transactions on Wireless Communications, vol. 3, no. 6, pp. 1918-1923, Nov 2004. doi: 10.1109/TWC.2004.837421

[67] R. Otnes and T. H. Eggen, "Underwater acoustic communications: Longterm test of turbo equalization in shallow water," IEEE Journal of Oceanic Engineering, vol. 33, no. 3, pp. 321-334, July 2008. doi: 10.1109/JOE.2008.925893

[68] K. Fang, L. Rugini, and G. Leus, "Low-complexity block turbo equalization for ofdm systems in time-varying channels," IEEE Transactions on Signal Processing, vol. 56, no. 11, pp. 5555-5566, Nov 2008. doi: 10.1109/TSP.2008.929129

[69] D. Anguita, D. Brizzolara, and G. Parodi, "Building an Underwater Wireless Sensor Network Based on Optical Communication: Research Challenges and Current Results," in Sensor Technologies and Applications, 2009. SENSORCOMM '09. Third International Conference on, 2009. doi: 10.1109/SENSORCOMM.2009.79 pp. 476-479.

[70] X. Che, I. Wells et al., "A static multi-hop underwater wireless sensor network using RF electromagnetic communications," in Distributed Computing Systems Workshops, 2009. ICDCS Workshops '09. 29th IEEE International Conference on, June 2009. doi: 10.1109/ICDCSW.2009.36 pp. 460-463.

[71] Y. Sun and T. Melodia, "The Internet Underwater: an IPCompatible Protocol Stack for Commercial Undersea Modems," in Proc. of ACM Intl. Conf. on UnderWater Networks and Systems (WUWNet), Kaohsiung, Taiwan, November 2013. doi: http://dx.doi.org/10.1145/2532378.2532407

[72] D. Pompili and I. Akyildiz, "A cross-layer communication solution for multimedia applications in underwater acoustic sensor networks," in Mobile Ad Hoc and Sensor Systems, 2008. MASS 2008. 5th IEEE International Conference on, 2008. doi: 10.1109/MAHSS.2008.4660052 pp. 275-284.

[73] — - "Overview of networking protocols for underwater wireless communications," Communications Magazine, IEEE, vol. 47, no. 1, pp. 97102, January 2009. doi: 10.1109/MCOM.2009.4752684

[74] —, "A Multimedia Cross-Layer Protocol for Underwater Acoustic Sensor Networks," Wireless Communications, IEEE Transactions on, vol. 9, no. 9, pp. 2924-2933, 2010. doi: 10.1109/TWC.2010.062910.100137

[75] U. Lee, P. Wang et al., "Pressure Routing for Underwater Sensor Networks," in INFOCOM, 2010 Proceedings IEEE, 2010. doi: 10.1109/INFCOM.2010.5461986 pp. 1-9.

[76] Y. Ren, W. K. G. Seah, and P. D. Teal, "Performance of pressure routing in drifting 3d underwater sensor networks for deep water monitoring," in Proceedings of the Seventh ACM International Conference on Underwater Networks and Systems, ser. WUWNet '12. New York, NY, USA: ACM, 2012. doi: 10.1145/2398936.2398972 pp. 28:1-28:8.

[77] Y. Noh, U. Lee et al., "VAPR: Void-Aware Pressure Routing for Underwater Sensor Networks," Mobile Computing, IEEE Transactions on, vol. 12, no. 5, pp. 895-908, 2013. doi: 10.1109/TMC.2012.53

[78] A. Goodney, Y. H. Cho et al., "An underwater communication and sensing testbed in Marina del Rey," in Proceedings of the Fifth ACM International Workshop on Underwater Networks (WUWNet), Woods Hole. Woods Hole, Massachusetts, USA: ACM, September 2010, p. 1.
[79] F. Guerra, P. Casari, and M. Zorzi, "World ocean simulation system (WOSS): A simulation tool for underwater networks with realistic propagation modeling," in Proceedings of the Fourth ACM International Workshop on UnderWater Networks, ser. WUWNet '09. New York, NY, USA: ACM, 2009. doi: 10.1145/1654130.1654134 pp. 4:1-4:8.

[80] J. Heidemann, M. Stojanovic, and M. Zorzi, "Underwater sensor networks: applications, advances and challenges," Philosophical Transactions of the Royal Society A: Mathematical, Physical and Engineering Sciences, vol. 370, no. 1958, pp. 158-175, 2012. doi: 10.1098/rsta.2011.0214

[81] F. Campagnaro, F. Favaro et al., "Simulation of multimodal optical and acoustic communications in underwater networks," in OCEANS 2015 . Genova, May 2015. doi: 10.1109/OCEANS-Genova.2015.7271518 pp. $1-7$.

[82] N. Meratnia, P. J. M. Havinga et al., "Clam - collaborative embedded networks for submarine surveillance: An overview," in OCEANS, 2011 IEEE - Spain, June 2011. doi: 10.1109/Oceans-Spain.2011.6003499 pp. $1-4$.

[83] L. Freitag, M. Grund et al., "A bidirectional coherent acoustic communication system for underwater vehicles," in OCEANS '98 Conference Proceedings, vol. 1, 1998. doi: 10.1109/OCEANS.1998.725794 pp. 482 486.

[84] J. Kojima, T. Ura et al., "High-speed acoustic data link transmitting moving pictures for autonomous underwater vehicles," in Underwater Technology, 2002. Proceedings of the 2002 International Symposium on, 2002. doi: 10.1109/UT.2002.1002439 pp. 278-283.

[85] C. Pelekanakis, M. Stojanovic, and L. Freitag, "High rate acoustic link for underwater video transmission," in OCEANS 2003. Proceedings, vol. 2, 2003. doi: 10.1109/OCEANS.2003.178494 pp. 1091-1097.

[86] H. Ochi, Y. Watanabe et al., "The acoustic communication experiment at 1,600 m depth using qpsk and 8psk," in OCEANS 2010, 2010. doi: 10.1109/OCEANS.2010.5664308 pp. 1-5.

[87] M. Tivey, P. Fucile, and E. Sichel, "A low power, low cost, underwater optical communication system," in Ridge 2000 Events, April 2004, pp. 27-29.

[88] F. Schill, U. R. Zimmer, and J. Trumpf, "Visible spectrum optical communication and distance sensing for underwater applications," in Australian Conf. on Robotics and Automation (ACRA), Canberra, Australia, December 2004.

[89] M. Doniec, I. Vasilescu et al., "AquaOptical: A Lightweight Device for High-Rate Long-Range Underwater Point-to-Point Communication," in OCEANS 2009, MTS/IEEE Biloxi - Marine Technology for Our Future: Global and Local Challenges, 2009, pp. 1-6.

[90] N. Farr, A. Bowen et al., "An integrated, underwater optical /acoustic communications system," in OCEANS 2010 IEEE. Sydney, Australia: IEEE, May 2010. doi: 10.1109/OCEANSSYD.2010.5603510 pp. 1-6.

[91] A. Palmeiro, M. Martin et al., "Underwater Radio Frequency Communications," in OCEANS, 2011 IEEE - Spain, 2011. doi: 10.1109/OceansSpain.2011.6003580 pp. 1-8.

[92] B. Li, S. Zhou et al., "Scalable ofdm design for underwater acoustic communications," in Acoustics, Speech and Signal Processing, 2008. ICASSP 2008. IEEE International Conference on, March 2008. doi: 10.1109/ICASSP.2008.4518857 pp. 5304-5307.

[93] M. Stojanovic, J. A. Catipovic, and J. G. Proakis, “Adaptive multichannel combining and equalization for underwater acoustic communications," The Journal of the Acoustical Society of America, vol. 94, no. 3, pp. 1621-1631, 1993. doi: http://dx.doi.org/10.1121/1.408135

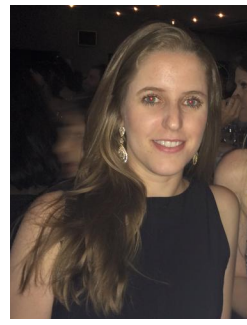

Camila M. G. Gussen was born in Brazil in 1986. She received the Electronics Engineering degree (cum laude) from the Federal University of Rio de Janeiro (UFRJ), Rio de Janeiro, Brazil, in 2009 and the M.Sc. degree in electrical engineering from COPPE/UFRJ in 2012. She is currently working towards the D.Sc. degree in electrical engineering at COPPE/UFRJ.

In 2011, she did an internship in Alcatel-Lucent Chair on Flexible Radio in Supélec, France. She also worked at Engenho - Pesquisa, Desenvolvimento e Consultoria ltda. Her research interests are in the fields of underwater acoustic communications, digital signal processing, digital communication, and wireless communications. 


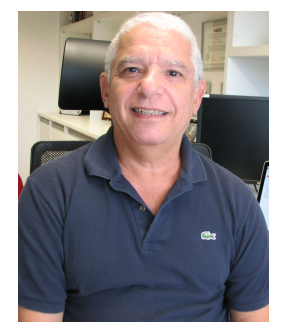

Paulo S. R. Diniz was born in Niterói, Brazil. $\mathrm{He}$ received the Electronics Eng. degree (Cum Laude) from the Federal University of Rio de Janeiro (UFRJ) in 1978, the M.Sc. degree from COPPE/UFRJ in 1981, and the Ph.D. from Concordia University, Montreal, P.Q., Canada, in 1984, all in electrical engineering.

Since 1979 he has been with the Department of Electronic Engineering (the undergraduate dept.) UFRJ. He has also been with the Program of Electrical Engineering (the graduate studies dept.) COPPE/UFRJ, since 1984, where he is presently a Professor. He served as Undergraduate Course Coordinator and as Chairman of the Graduate Department. He has received the Rio de Janeiro State Scientist award, from the Governor of Rio de Janeiro state.

From January 1991 to July 1992, he was a visiting Research Associate in the Department of Electrical and Computer Engineering of University of Victoria, Victoria, B.C., Canada. He also held a Docent position at Helsinki University of Technology (now Aalto University). From January 2002 to June 2002, he was a Melchor Chair Professor in the Department of Electrical Engineering of University of Notre Dame, Notre Dame, IN, USA. His teaching and research interests are in analog and digital signal processing, adaptive signal processing, digital communications, wireless communications, multirate systems, stochastic processes, and electronic circuits. He has published several refereed papers in some of these areas and wrote the text books ADAPTIVE FILTERING: Algorithms and Practical Implementation, Fourth Edition, Springer, NY, 2013, and DIGITAL SIGNAL PROCESSING: System Analysis and Design, Second Edition, Cambridge University Press, Cambridge, UK, 2010 (with E. A. B. da Silva and S. L. Netto), and the monograph BLOCK TRANSCEIVERS: OFDM and Beyond, Morgan \& Claypool, New York, NY, 2012 (W. A. Martins, and M. V. S. Lima)

He has served as the Technical Program Chair of the 1995 MWSCAS held in Rio de Janeiro, Brazil. He was the General co-Chair of the IEEE ISCAS2011, and Technical Program co-Chair of the IEEE SPAWC2008. He has also served Vice President for region 9 of the IEEE Circuits and Systems Society and as Chairman of the DSP technical committee of the same Society. $\mathrm{He}$ is also a Fellow of IEEE and EURASIP. He has served as associate editor for the following Journals: IEEE Transactions on Circuits and Systems II: Analog and Digital Signal Processing from 1996 to 1999, IEEE Transactions on Signal Processing from 1999 to 2002, and the Circuits, Systems and Signal Processing Journal from 1998 to 2002 . He was a distinguished lecturer of the IEEE Circuits and Systems Society for the year 2000 to 2001. In 2004 he served as distinguished lecturer of the IEEE Signal Processing Society and received the 2014 Charles Desoer Technical Achievement Award of the IEEE Circuits and Systems Society. He also holds some best-paper awards from conferences and from an IEEE journal. He is currently serving as a Regional Director of the IEEE Signal Processing Society.

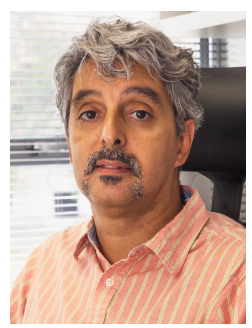

Marcello L. R. Campos was born in Niteroi, Brazil, in 1968. He received the Engineering degree (cum laude) from the Federal University of Rio de Janeiro (UFRJ), Rio de Janeiro, Brazil, in 1990, the M.Sc. degree from COPPE/UFRJ in 1991, and the Ph.D. degree from the University of Victoria, Victoria, BC, Canada, in 1995, all in Electrical Engineering.

In 1996, he was post-doctoral fellow with the Department of Electronics, School of Engineering, UFRJ, and with the Program of Electrical Engineering, COPPE/UFRJ. From January 1997 until May 1998, he was Associate Professor with the Department of Electrical Engineering (DE/3), Military Institute of Engineering (IME), Rio de Janeiro. He is currently Professor of the Program of Electrical Engineering, COPPE/UFRJ, where he served as Department Vice-Chair and Chair in the years 2004 and 2005, respectively. From September to December 1998, he was visiting the Laboratory for Telecommunications Technology, Helsinki University of Technology, Espoo, Finland. In 2001, he received a Nokia Visiting Fellowship to visit the Centre for Wireless Communications, University of Oulu, Oulu, Finland. In 2008, he visited Unik, the University Graduate Center of the University of Oslo, Oslo, Norway, and in 2016 he visited Aalto University, Helsinki, Finland, as part of the mobility program for Smart ${ }^{2}$ project, Erasmus Mundus Programme.
Prof. Campos served as IEEE Communications Society Regional Director for Latin America in 2000 and 2001, Local-Arrangements Co-Chair for GLOBECOM'99, Finance Chair for SPAWC 2008, Plenary Chair for ISCAS 2011, and Technical Co-Chair for the 2013 Brazilian Telecommunications Symposium. He founded and is the current Chair of the IEEE Signal Processing Society Rio de Janeiro Chapter. He has taught over 150 courses on mobile communications in 15 countries, and his research interests include adaptive signal processing in general and its application to distributed networks in particular, adaptive beamforming, statistical signal processing, signal processing for communications, underwater, mobile and wireless communications, and MIMO systems.

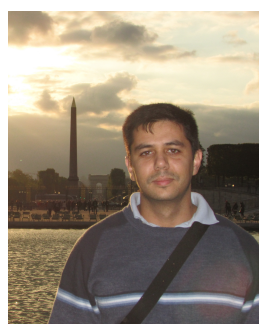

Wallace A. Martins was born in Brazil in 1983. He received the Electronics Engineer degree from the Federal University of Rio de Janeiro (UFRJ) in 2007, the M.Sc. and D.Sc. degrees in Electrical Engineering from COPPE/UFRJ in 2009 and 2011, respectively. In 2008, he was a research visitor at the Department of Electrical Engineering, University of Notre Dame, Notre Dame, IN. In 2016, he was a research visitor at Université Lille 1, Lille, France. Since 2013 he has been with the Department of Electronics and Computer Engineering (DEL/Poli) and Electrical Engineering Program (PEE/COPPE), UFRJ, where he is presently an Associate Professor. His research interests are in the fields of digital communications, underwater communications, microphone/sensor array processing, adaptive signal processing, and machine learning techniques. Dr. Martins received the Best Student Paper Award from EURASIP at EUSIPCO2009, Glasgow, Scotland, and the 2011 Best Brazilian D.Sc. Dissertation Award from Capes.

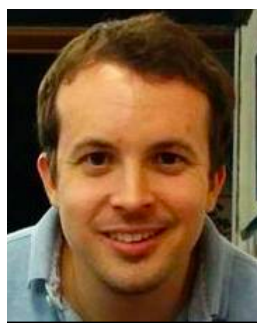

Felipe M. Costa obtained his bachelor degree in communications and networking engineering from University of Brasilia, in Brazil in 2007 and his master's degree in electrical and computer engineering from Yokohama National University, in Japan in 2010. From 2010 until 2012, he worked for Nokia Institute of Technology in Brazil, where he used to develop cellular network simulators. In 2012, Felipe joined GE Global Research Center in Rio de Janeiro, Brazil, where he developed research and projects related to data communications. Felipe currently is an entrepreneur and is launching his startup named Cloudia in São Paulo, Brazil.

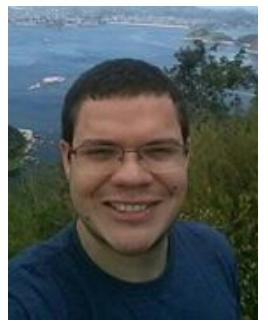

Jonathan N. Gois was born in Rio de Janeiro, Brazil, in 1990. He has received the Electronics engineering degree from Universidade Federal do Rio de Janeiro in 2013 and the M.Sc. degree in Electrical Engineering, in 2016, from the same university. Since 2016, he is a Department of Electrical Engineering's Professor at Centro Federal de Educação Tecnológica Celso Suckow da Fonseca, in Rio de Janeiro, Brazil. His research interests include image processing, video processing, video fusion, machine learning and subsea communications. 